\title{
PYCNOGENOL: A NUTRACEUTICAL FOR VENOUS HEALTH
}

\author{
Om P. Gulati \\ Horphag Research Management Ltd., Geneva, Switzerland
}

Recent studies utilizing highly sophisticated methodology and techniques were able to provide a new insight into the molecular mechanism of the biological activity of various botanical extracts. The present review provides an update of the multifaceted biological profile of the nutraceutical Pycnogenol ${ }^{\circledR}$ in venous health, using a target oriented approach in light of pathophysiology of chronic venous insufficiency. Pycnogenol is French maritime pine bark extract produced by extraction of the outer bark of Pinus pinaster Ait. Subsp. atlantica. Pycnogenol has strong antioxidant profile proven by in vitro and in vivo studies in animals and further confirmed in clinical trials. Its strong antioxidant profile, vasodilator activity, antithrombotic effect and its collagen stabilizing property make it a unique health product. It reduces edema of legs and lowers chances of developing deep venous thrombosis and skin ulceration. These properties further qualify Pycnogenol to be a useful food supplement for venous health particularly in chronic venous insufficiency. Developing new combination products using synergistic approaches are the future trends of research and development to prove efficacy in conditions which involves oxidative stress, inflammation, endothelial dysfunction, thrombogenesis, and ulceration. Biomed Rev 2008; 19: 33-43.

Key words: chronic venous insufficiency, food supplement, nutrigenomics

\section{INTRODUCTION}

In the recent years, a paradigm shift in the nutritional science has been evolved, nutrition research being targeted to molecular biology, genetics and adipobiology. In essence, the sequencing of mammalian genomes has initiated the development of novel methodologies to study gene expressions for as many genes as possible, simultaneously. Additionally, transcriptomics, proteomics, interactomics and metabolomics have opened new areas of science, including nutrigenomics (nutritional genomics) and pharmacogenomics (1). Hence neutraceuticals (food- and botanic-derived products) have been emerging as a promising innovative approach for maintaining good health and reducing the risk of various diseases.

The present review provides an update of the multifaceted biological profile of the nutraceutical (botanical) Pycnogenol ${ }^{\circledR}$ in venous health, using a target oriented approach in light of pathophysiology of chronic venous insufficiency (CVI).

\section{PYCNOGENOL}

Pycnogenol is French maritime pine bark extract produced by extraction of the outer bark of Pinus pinaster Ait. Subsp. atlan-

Received 17 November 2008, accepted 15 December 2008.

Correspondence and reprint request to Dr Om P. Gulati, Horphag Research Management Ltd., Avenue Louis-Casai 71, 1216

Geneva, Switzerland. Tel.: 4122710 2650, Fax: 4122710 2600, E-mail: om@horphag.com 
tica. Its specifications are described in the USP 30 - Dietary supplements (2). On safety aspects, it is generally recognized as safe (GRAS) in the USA (3). Pycnogenol has strong antioxidant and anti-inflammatory activities (4).

The historical development of Pycnogenol and the utilization of the pine bark as a health promoting botanical (5) and its role in cardiovascular health and blood glucose control has been described earlier (6). The concept of orally administered Pycnogenol, either as "stand alone product" or in combination with other food ingredients was developed during last two decades. Numerous clinical trials have investigated the efficacy of oral Pycnogenol in individuals with CVI (7-14), clinical research data being reviewed (6,15-17).

\section{PATHOPHYSIOGY OF CHRONIC VENOUS INSUFFICIENCY}

Chronic venous insufficiency is characterized by valvular incompetence of the perforator veins, venous stasis, resulting in venous hypertension and increase in venular permeability leading to edema of the legs and feet (18-20). The six most important manifestations involved in CVI are $(i)$ venous stasis and hemodynamic alterations, (ii) endothelial dysfunction, (iii) edema formation, (iv) collagen destabilization, (v) microcirculatory dysfunction, and (vi) leukocyte-endothelial interactions.

\section{Venous stasis and hemodynamic alterations}

During venous stasis, vasomotion frequency in CVI patients decreased significantly from $7.4 \pm 2.2 \mathrm{cpm}$ to $5.4 \pm 2.5 \mathrm{cpm}$, whereas vasomotion frequency in control participants increased significantly from $4.5 \pm 1.6 \mathrm{cpm}$ to $8.8 \pm 2.5 \mathrm{cpm}$. The failure to increase vasomotion frequency in CVI is considered to reflect longer dilatation of the distal venous vessels per time unit and increase in hematocrit (21). Continuous long hours sitting or standing prolonged bending of legs, pregnancy, overweight or obesity lead to venous stasis, increase in hydrostatic pressure in the blood vessels, decrease in venous flow and increase interstitial osmotic pressure. These events lead to increased infiltration of fluid from intravascular compartment to extravascular compartment leading to augmentation of legs and feet edema. Oxidative stress plays a great role in venous stasis and CVI. A statistically significant increase of venous pressure of the lower limbs was observed in workers with standing occupation (surgery room nurses) associated with significantly higher mean levels of reactive oxygen species (ROS) (22). These phenomena have also been demonstrated in experimental CVI in rats (23) Fluorescence angiography technique was used to evaluate the haemodynamic changes and these were further confirmed using radioactive microspheres technique $(23,24)$.

\section{Endothelial dysfunction, edema, and microcirculatory dysfunction}

Edema of feet and legs is one the most frequent signs of CVI. Damage of endothelium is particularly the first relevant event in its pathogenesis. Altered vascular permeability and endothelial dysfunction also lead to thrombosis, the well recognized and most frequent sign of CVI. These phenomena have earlier been demonstrated by simulating an experimental models of CVI and of dextran and D-galactosamine-induced edema in rats (23-25). The extent of edema depends on the degree of damage caused to the endothelium, the extent of accumulation of leukocytes, and the degranulation of mast cells releasing histamine (26-28), accompanied by collagen destabilization (29).

Chronic venous insufficiency is associated not only with functional and morphologic changes of the deep veins but also the microcirculation. In CVI, changes in skin capillaries are stage dependent. There is a decrease of capillary density from 27 capillaries per $\mathrm{cm}^{2}$ down to 5 capillaries per $\mathrm{cm}^{2}$ in CVI stage III as classified by Widmer (30). Edema is resulting from capillary leakage as shown by perfusion experiments with fluorescent dyes (31). The transcutaneous partial pressure of oxygen $\left(\mathrm{TcPO}_{2}\right)$ is about $57 \mathrm{~mm} \mathrm{Hg}$ in healthy individuals, and decreased stage-dependent in CVI is less than $30 \mathrm{~mm} \mathrm{Hg}$ in stage III Widmer classification. A lack of oxygen is unlikely to be primary reason for the development of skin ulcers in CVI (32). Impairment of the cutaneous microcirculation is a major risk factor in inflammation and ulceration in patients with CVI. Increase of capillary filtration rate predisposes to the formation of edema (33). Further leukocyte-endothelial interactions and ongoing inflammation can be found in CVI, including the expression of leukocyte adhesion molecules (34); these might be new targets for pharmacologic intervention in CVI (35)

\section{BIOLOGICAL PROFILE OF PYCNOGENOL}

\section{Antioxidant and anti-inflammatory activities}

Antioxidant and anti-inflammatory profile of Pycnogenol has been reviewed (36-39); these biological activities are shown in vitro and in vivo models and then have been confirmed in clinical studies. These include: (i) antioxidant and free radical scavenging activity (40-44), (ii) antioxidant activity sparing vitamin $\mathrm{C}$ and recycling of vitamin $\mathrm{E}$ (45), (iii) inhibition of lipid peroxidation (46), (iv) protection of nerve cells against $\beta$-amyloid- or glutamate-induced toxicity (47), (v) erythrocytes 
protection in G6PD deficient human (48), (vi) inhibition of generation of inflammatory and oxidative mediators (49-55), (vii) inhibition of matrix metalloprotease activity (56), (viii) inhibition of histamine release from mast cells (57), and (ix) wound healing effects $(58,59)$.

\section{Antioxidant profile: In vitro studies}

Several studies made with Pycnogenol have been reported to demonstrate its free radical scavenging and/or antioxidant activity in vitro. Free radical (hydroxyl and superoxide) scavenging activity was measured using a highly sensitive electron spin resonance spectrometer and was compared to other bioactive free radical scavengers like ginkgo biloba and green tea extract. An analogue of vitamin C and vitamin E were used as reference standards for hydroxyl radicals. Superoxide dismutase (SOD) was used as the reference standard for superoxide anion scavenging activity. Macrophages were activated by the bacterial wall components and lipopolysaccharides (LPS) and interferon- $\gamma($ IFN- $\gamma$ ), which induces the expression of large amounts of the enzyme nitric oxide synthase (iNOS). Pycnogenol was found to a potent free radical scavenger of hydroxyl, superoxide and NO radicals $(40,41)$. Pycnogenol participates in the cellular antioxidant network as indicated by its ability to regenerate the ascorbyl radical and to protect endogenous vitamin $\mathrm{E}$ and glutathione from oxidative stress. In addition, it was found to be resistant to the action of heat and ascorbate oxidase (45). Pycnogenol protects DNA against Fenton reaction radicals, probably by chelating Fe. It also can induce SOD under oxidative stress (46). In human umbilical vein endothelial cell cultures, Pycnogenol exhibited a dosedependent suppression of tumor necrosis factor-alpha (TNF$\alpha$ )-induced activation of the transcriptional regulatory protein nuclear factor-kappa B (NF-kB). Expression of cell surface molecules such as VCAM-1 and ICAM-1 was reduced (47).

In two different in vitro studies, bovine vascular endothelial cells were treated with Pycnogenol before subjecting them to oxidative stress induced by t-butyl hydroperoxide (t-HBP), malondialdehyde (MDA) being used as a biological marker to assess lipid peroxidation. Preincubation of endothelial cells with Pycnogenol at concentrations $10-80 \mu \mathrm{g} / \mathrm{ml}$ for 16 $\mathrm{h}$ increased the cell viability after t-BHP treatment and, in addition, caused a dose-dependent decline in $\operatorname{MDA}(45,46)$.

In another independent in vitro study model, bovine retina as the tissue substrate and lipid peroxidation as the target reaction action giving rise to lipid hydroperoxide as the biological marker expressed as thiobarbituric acid reactive substances
(TBARS), Pycnogenol effectively inhibited lipid peroxidation at a concentration as low as $25 \mathrm{ng} / \mathrm{ml}$. Lipid peroxidation was inhibited by Pycnogenol in a dose-dependent fashion and was completely absent at a concentration of $250 \mathrm{ng} / \mathrm{ml}$. Pycnogenol was relatively more effective than grape seed extract, vitamin C, vitamin E, and lipolic acid (44).

Antioxidant activity of Pycnogenol was further confirmed by using three different in vitro models addressing the oxidative burst, LDL oxidation and iron/ascorbic acid system as oxidant challenges on different substrates. Pycnogenol exhibited a concentration-dependent inhibition of oxidative burst triggered by zymosan in J774 murine macrophages in vitro. Pycnogenol when coincubated with copper sulphate used to oxidise human plasma LDL (formation of thiobarbituric acid reactive substances, TBARS used as markers) resulted in inhibition of LDL oxidation in a concentration dependent manner. Pycnogenol significantly minimised the cleavage of DNA caused by hydroxyl radical induced by exposure of pBR322 plasmid DNA to iron/ascorbic acid system and measured by agarose gel electrophoresis (43).

Pretreatment of LPS stimulated murine macrophages (RAW 264.7) associated with increased release of the proinflamatory mediators interleukin-1 $\beta$ (IL-1 $\beta$ ) and TNF- $\alpha$. Incubation with Pycnogenol was associated with a dose-dependent decrease in these mediators' levels, which blocks the activation of two major "proinflammatory" transcription factors, NF- $\mathrm{kB}$ and AP-1 (47).

\section{Antioxidant and anti-inflammatory profile: In vivo animal studies}

Pycnogenol was shown to have remarkable free radical scavenging activity in vitro and anti-inflammatory activity in vivo. These activities bear close correlation indicating the involvement of free radicals in inflammation and the antiinflammatory action of Pycnogenol at least partly due to its free radical scavenging effect (40). Anti-inflammatory and wound healing effects were demonstrated subsequently by the same group of authors $(53,54,58)$.

There is enough experimental evidence that oxidative stress is involved in the pathophysiology of diabetes and its complications. In streptozotocin-induced diabetic rats the glutathione to glutathione disulfide ratio and the activities of endogenous antioxidant enzymes superoxide dismutase, catalase, glutathione peroxidase, glutathione reductase, and $\gamma$ glutamyl-transpeptidase were significantly increased after Pycnogenol administration. These changes were associated with a significant decrease in blood glucose levels in diabetic 
rats (60). Another study from the same laboratory further showed that Pycnogenol administered alone or in combination with $\beta$ carotene, once again increased glutathione reductase activities (61).

The experiments were repeated focusing on diabetic retinopathy in streptozotocin-induced diabetic rats. Decreased retinal $\gamma$-glutamyl transferase activity of diabetic rats was normalized by administration of Pycnogenol alone or in combination with $\beta$-carotene. Elevated activity of SOD in diabetic retina was normalized by Pycnogenol and $\beta$-carotene combination (62).

\section{Clinical research}

The results of the clinical studies showed the efficacy of Pycnogenol in patients with CVI and other venocapillary diseases confirming that oxidative stress is important in venous diseases and that an antioxidant dietary supplementation with Pycnogenol may influence the positive outcome of the CVI. The results are consistent with the proposed mechanism of action of Pycnogenol: venous blood flow is not modified, while a reduced capillary permeability is observed. The reduction of the subcutaneous edema and, consequently, of the subjective feeling of heavy legs might be the result of free radical scavenging activity of Pycnogenol.

Clinical research data on Pycnogenol are provided based on its antioxidant activity in healthy volunteers. The effect of Pycnogenol on human antioxidant defenses was demonstrated by a significant $(p<0.05)$ decrease of oxygen radical absorbance capacity in plasma throughout the Pycnogenol supplementation period of three weeks. In addition to its ability to enhance plasma antioxidant capacity, Pycnogenol significantly reduced LDL-cholesterol levels and increased HDL-cholesterol levels in the blood (63). In another independent double-blind study, Pycnogenol significantly increased plasma antioxidant activity $(p<0.01)$. The level of total cholesterol decreased from 5.41 to $4.98 \mathrm{mmol} / \mathrm{L}$ associated with a decrease LDL-cholesterol from 3.33 to $2.78 \mathrm{mmol} / \mathrm{L}(64)$.

After oral application of Pycnogenol, two major metabolites are formed in vivo, delta-(3,4-dihydroxyphenyl)-gammavalerolactone (M1) and delta-(3-methoxy-4-hydroxyphenyl)gamma-valerolactone (M2). Both metabolites exert strong inhibitory effects on matrix metalloproteinases types 1,2 and 9. M1 is also reported to have superoxide scavenger activities (56).

An acute exposure to ultraviolet radiation (UVR) leads to inflammatory response, skin erythema. Oxidative stress by releasing ROS and reactive nitrogen species (RNS) is involved in producing this biological effect. UVR stimulates expression of the proinflammatory genes for TNF- $\alpha$, IL- $1 \alpha$, IL-1 $\beta$, IL-6, and IL-8. All these cytokines/chemokines contain NF- $\mathrm{BB}$ binding sites in the 5 'flanking region of the gene.

Pycnogenol produced a significant increase in the dose of UVR necessary to achieve standardized erythema response (minimum erythema dose) of human skin. The activation of NF- $\kappa B$ plays a major role in the UVR-induced erythema. Pycnogenol inhibited UVR-induced NF- $\mathrm{kB}$-dependent gene expression in a concentration dependent manner (51).

Oxidative stress is also involved in pathogenesis of other clinical conditions like skin ageing, erythema, abnormal sperm morphology, and gingival bleeding and plaque formation. The effects of Pycnogenol were studied independently in these conditions. Supplementation with Pycnogenol along with other micronutrients in a formulation Evelle improved visible signs of skin aging, increased skin elasticity and decreased skin roughness (65). Pycnogenol supplementation provided relief from erythema and melasma (51,66). Additionally, Pycnogenol by virtue of its antioxidant profile has improved abnormal sperm morphology and functions (67), provided relief from pain in dysmenorrhea (68) and minimized gingival bleeding and plaque formation (69).

Fifteen clinical studies have been performed between 1989 and 1999 in 784 patients suffering from CVI and other venous diseases (reviewed in 15). The results of the clinical studies show the efficacy of Pycnogenol in patients with CVI and other veno-capillary diseases. They confirm that oxidative stress plays an important role in venous diseases and the antioxidant dietary supplement Pycnogenol may influence the healthy outcome of CVI. The results are consistent with the proposed mechanism of action of Pycnogenol: venous blood flow is not modified, while a reduced capillary permeability is observed: the reduction of edema and consequently, of subjective feeling of heavy legs might be the result of free radical scavenging activity of Pycnogenol and reduced leukocytes adhesion and migration in the extravascular spaces. The sealing effect on capillaries results from strong tissue binding of Pycnogenol leading to a cross linking of proteins of the vessel wall, which may contribute to reduction in edema formation.

\section{Anti-edema effect in chronic venous insufficiency}

A double-blind placebo-controlled clinical study was performed to investigate the efficacy of Pycnogenol in CVI. Forty patients with CVI and varices of the legs were randomly assigned to a treatment with Pycnogenol, $100 \mathrm{mg}$ x 3/day or a 
placebo for 2 months. The effects were evaluated by scoring the symptomatology with a semi-quantitative scale, and the venous blood flow by means of a hand-held Doppler ultrasound (venous blood flow meter). Pycnogenol supplementation produced a significant reduction in subcutaneous edema as well as heaviness and pain in the legs, on both after 30 and 60 days, the evaluation time periods. Approximately $60 \%$ of patients treated with Pycnogenol experienced a complete disappearance of edema (the most rapidly disappearing symptom) and pain at the end of supplementation, while almost all the patients reported a reduction in leg heaviness which disappeared in approximately $33 \%$ of patients. These changes were statistically significant. No effect was observed in the placebo-treated subjects. No effect on the venous blood flow was observed in either of the experimental groups (7). The results were confirmed in a subsequent clinical study. This study was performed in two phases. First phase consisted of a double-blind phase - in which 20 patients were recruited and randomly treated with placebo or Pycnogenol (100 mg 3/day for 2 months), and second phase represented an open phase - in which other 20 patients were supplemented with Pycnogenol at the same dose schedule. In total, 40 patients were enrolled; 30 of them were treated with Pycnogenol and 10 with placebo. Pycnogenol significantly improved the legs' heaviness and subcutaneous edema; the venous pressure was also significantly reduced by this nutraceutical. Clinically, capillary leakage, perivascular inflammation and subcutaneous edema were all reduced (8).

The above two studies were followed by a comparative clinical study, where efficacy of food supplement Pycnogenol was compared to standard drug Venostasin (horse chestnut seed extract) in CVI. 40 patients with diagnosed CVI were administered either $600 \mathrm{mg}$ Venostasin or $360 \mathrm{mg}$ Pycnogenol per day over a period of 4 weeks. The following parameters were investigated before the start and after 2 and 4 weeks of treatment: circumference of the lower legs and rating of subjective symptoms (scores) of pain, cramps, night-time swelling, feeling of "heaviness", and reddening of the skin. In addition, blood levels of cholesterol LDL and HDL were determined before and at the end of treatment. Pycnogenol significantly reduced the circumference of the lower limbs and significantly improved subjective symptoms. Furthermore, it significantly decreased LDL-cholesterol values in the blood, whereas HDL-cholesterol remained unaffected. Venostasin only moderately but not significantly, reduced the circumference of the lower limbs and marginally improved symptoms. Venostasin had no influence on the determined lipid values.
In conclusion, Pycnogenol was found to be more efficacious than Venostasin (9).

In a study with 18 patients suffering from venous malleolar ulcers, Pycnogenol was applied orally or orally and topically for 6 weeks. Ulcer healing was faster in the combination group, but both treatment groups showed a reduction of the permalleolar edema. This was interpreted as an improvement of local microcirculation (59).

\section{Effects on venous tone}

A placebo-controlled double-blind study with Pycnogenol (360 $\mathrm{mg}$ /day for 5 days) in 40 patients suffering from venous circulation problems is reported. Its efficacy has been confirmed on the basis of objective and subjective signs and symptoms of static edema. The continuous sitting for 2 hours was used to induce stasis edema. Objective evaluation parameter was swelling of leg: lower leg volume was measured using the method of Artzler and Herbst with modified apparatus (water displacement technique. Subjective evaluation parameters were signs and symptoms, using 5-point scores. These were heaviness, pain, itching and night cramp. The comparative volume measurement after 2 hours sitting position revealed impressive differences between Pycnogenol treated group and placebo group. Whereas the resting volume was somewhat higher in the placebo group patients at the end of treatment, these values significantly decrease in Pycnogenol treated group $(\mathrm{p}<0.01)$. In a placebo-controlled double blind study in 29 patients, it was possible to detect a significant reduction ( $\mathrm{p}<$ 0.01 ) of venous stasis (sitting for 1 and 2 hours respectively) oedema after 4 days treatment with Pycnogenol at a dose 180 $\mathrm{mg}$ /day in comparison to placebo (11).

\section{Anti-edema effect in healthy subjects during long flights}

The anti-edema effects of Pycnogenol was evaluated during long-haul flights in asymptomatic subjects. The assessment of edema was performed by evaluating an analogue scale, the rate of ankle swelling by strain-gauge derived rate of ankle swelling (RAS), and by assessing the ankle circumference variation. The study included 211 subjects; 169 completed the study ( 88 in the control group and 81 in the Pycnogenol group). There were no important differences between the two groups (comparable for age, gender, weight, body mass index, and pattern distribution). The oedema score, the RAS, and the circumference at inclusion were also comparable. After the flight, in subjects treated with Pycnogenol the oedema score 
was increased only by $17.9 \%$ (vs. an increase of $58.3 \%$ in the control group) $(\mathrm{p}<0.05)$. The RAS, evaluated in 22 subjects in the Pycnogenol group (age 44.5; SD 8) and in 23 in the control group (age 45; SD 9) was increased on average by $91 \%$ in the control group and $36 \%$ in the Pycnogenol group ( $<<0.05$ ). The variation on circumference at the ankle was $6 \%$ in the Pycnogenol group ( $11 \%$ in the control group; $\mathrm{p}<0.05)$. These results indicate a positive effect of Pycnogenol on oedema during long flights when considering subjective and objective data. No unwanted effects were observed $(70,71)$.

\section{Microcirculatory function}

A clinical study was performed to investigate the clinical efficacy of oral Pycnogenol in patients with severe CVI in comparison was the combination of diosmin and hesperidin Daflon. A group of 86 patients with severe CVI, venous hypertension, ankle swelling and previous history of venous ulcerations received either oral Pycnogenol (capsules) 150 $\mathrm{mg}$ or $300 \mathrm{mg}$ daily for 8 weeks or Daflon, 1,000 mg/day. At the end of the study, microcirculatory results indicated: $(i)$ a progressive decrease of skin flux at rest (RF), (ii) a significant decrease in capillary filtration, (iii) an improvement in the symptomatic venous score, (iv) edema reduction, and (v) a significant improvement (increase) in $\mathrm{pO}_{2}$ and a decrease in $\mathrm{pCO}_{2}$ in the Pycnogenol group.

A significant level of improvement was reached after 4 weeks of treatment in most patients $(\mathrm{p}<.05)$ of the Pycnogenol group while clinical improvement was significant only in 6 subjects in the Daflon group. The positive effects of treatment with Pycnogenol after 8 weeks were significantly more important in comparison with the Daflon group. In conclusion, this study confirms the fast clinical efficacy of Pycnogenol in patients with chronic venous insufficiency and venous microangiopathy and its superiority - considering the evaluated parameters - to the combination of diosmin and hesperidin (13).

Microscopic evaluation of microvessels at the root of the finger's nails showed that consumption of $60 \mathrm{mg}$ Pycnogenol per day for 4 weeks significantly dilate these vessels and improve microcirculation. After discontinuation of Pycnogenol the improved microcirculation persists for up to 4 weeks. When Pycnogenol tablets were chewed an increased microcirculatory blood flow was observed in $78 \%$ of the subjects (72).

\section{Improvement in cramps and muscular pain}

A clinical study was performed to assess the action of Pycnogenol on cramps and muscular pain in different groups of subjects including patients with CVI. Cramps in legs are very common in CVI. The study included a 5-week observation period (4 weeks treatment and one follow-up week after the suspension of treatment) to evaluate the efficacy of Pycnogenol after its withdrawal. Four $50 \mathrm{mg}$ capsules (total dose $200 \mathrm{mg} /$ day) were prescribed with suggestion to drink at least 1.5 liters of water every day. In the first part of the study 66 healthy subjects completed a 5-week follow-up period. The difference between number of cramps attacks recorded within the 2 weeks before inclusion and the number of episodes during the fourth $(\mathrm{p}<0.05)$ and fifth $(\mathrm{p}<0.05)$ week were statistically significant. In normal subjects the average number of episodes was reduced from 4.8 (1.2) events per week to $1.3(1.1)$ at 4 weeks $(\mathrm{p}<0.05)$. In venous patients the decrease in events was from $6.3(1.1)$ to $2.6(0.4)$ per week $(\mathrm{p}<0.05)$. In athletes the number of episodes decreased from 8.6 (2) to $2.4(0.5)(\mathrm{p}<0.05)$. The decrease was still present at 5 weeks in the 3 groups, to levels significantly lower than inclusion values $(\mathrm{p}<0.05)$. There was a significant decrease in the number of cramps episodes ( $p$ $<0.05)$ and in the score concerning muscular pain $(\mathrm{p}<0.05)$ in claudicants and diabetics. No significant effects were observed in the placebo groups. Altogether, these results suggest that the use of Pycnogenol prevents cramps, muscular pain at rest, and pain after/during exercise in normal, in athletes prone to cramps, and in patients with venous disease (14).

\section{Antithrombotic effect in healthy subjects during long flights}

A clinical study was performed to evaluate the occurrence of deep venous thrombosis (DVT) and superficial vein thrombosis (SVT) and its prophylaxis with Pycnogenol, in longhaul flights, in subjects at moderate to high-risk of DVT and SVT. The study pre-included 244 pre-selected subjects; 211 were included ( 33 were excluded for several reasons due to logistic problems) and 198 completed the study; 13 subjects were lost for follow-up at the end of the flight, all for nonmedical problems (i.e., for difficult connections). All subjects were scanned within 90 minutes before the flight and within 2 hours after disembarking. Subjects were supplemented with $100 \mathrm{mg}$ Pycnogenol per capsule. Treatment subjects received two capsules between 2 and 3 hours before flights with 250 $\mathrm{mL}$ of water; two capsules were taken 6 hours later with 250 $\mathrm{mL}$ of water and one capsule the next day. The control group received comparable placebo at the same intervals. The flight duration was on average 8 hours and 15 minutes (SD $55 \mathrm{~min}$ ) (range 7.45-12.33). In the control group there were five thrombotic events (one DVT and four superficial thromboses) while 
only non thrombotic, localized phlebitis was observed in the Pycnogenol group (5.15\% vs. no events; $\mathrm{p}<0.025)$. The ITT (intention to treat) analysis detects 13 failures in the control group (eight lost to follow up + five thrombotic events) of 105 subjects (12.4\%) vs. five failures (4.7\%; all lost, no thrombotic events) in the treatment group $(\mathrm{p}<0.025)$. No unwanted effects were observed. In conclusion, this study indicates that Pycnogenol treatment was effective in decreasing the number of thrombotic events (DVT and SVT) in moderate-to-high risk subjects, during long-haul flights.

Based on antithrombotic effect of Pycnogenol, a rationale to develop a concept of another combination product with Pycnogenol and Ginger extract was developed and realized to give rise to a new formulation Zinopin (73) which was clinically tested in long halt flights for its efficacy on thrombotic events and motion sickness (74).

\section{Healing of venous ulcers}

The above findings were applied to a clinical situation in a double blind clinical study performed in patients with CVI. Chronic venous insufficiency causes a well-defined microangiopathy described as venous hypertensive microangiopathy (VHM) leading to venous ulcerations, which is mainly observed in the distal part of the leg. In VHM edema is the consequence of increased capillary pressure and reduced local clearance, and this affects local perfusion. The healing of venous ulcers is usually very slow. Oral Pycnogenol is effective in venous disease and particularly in controlling edema. The aim of this study was the evaluation of the local effects of Pycnogenol on ulcers healing associated with venous hypertension. The study lasted 6 weeks including 18 patients (16 completed the study) with venous ulcerations. The oral treatment with Pycnogenol was compared to a combination treatment including oral and local treatment. In subjects treated with the combination treatment (oral and local), venous ulcers healed better (there was a faster reduction in ulcerated area) in comparison with oral treatment only. According to this pilot study Pycnogenol appears to have an important role in reducing the risk of venous ulcers improving their healing (59).

A subsequent double blind clinical study was performed to investigate the Clinical efficacy of oral Pycnogenol in patients with severe chronic venous insufficiency Patients received oral Pycnogenol (50 mg capsules, 3 times daily for a total of $150 \mathrm{mg}$ daily) for 8 weeks. A group of 21 patients was included in the Pycnogenol group and 18 equivalent patients were observed as controls. All 21 patients (age 53 years; range, 42-60 years;
$\mathrm{M}: \mathrm{F}=11: 10$ ) in the Pycnogenol group completed the 8-week study period. In all supplemented subjects, microcirculatory and clinical evaluations indicated a progressive decrease in skin influx, indicating an improvement in the level of microangiopathy: a significant decrease in capillary filtration; a significant improvement in the symptomatic score; and a reduction in edema. Pycnogenol supplementation may thus reduce the risk of ulcerations by controlling the level of venous microangiopathy (75) .

\section{Pycnogenol decreases serum thromboxane levels and thus inhibits thrombogenesis}

In smokers, thromboxane A2 (TxA2) released from platelets gets converted to thrombxane B2 (TXB2), a powerful vasoconstrictor. Smoking induces increased in circulating levels of TxB2. In a clinical study performed in smokers, Pycnogenol administration at a dose of $200 \mathrm{mg} /$ day lowered TxB2 levels to those of non-smokers, whereas showing no effect on the already low levels of thromboxane B2 in nonsmokers. In effect, Pycnogenol supplementation reduces a risk factor for venous thrombosis (76). These findings were confirmed in another independent double-blind, randomized, cross-over study in 11 human subjects with high blood pressure it was shown that Pycnogenol administered at a daily dose of $200 \mathrm{mg}$ for 8 weeks significantly $(\mathrm{p}<0.05)$ decreased serum TxB2 in serum from baseline $151 \pm 4 \mathrm{ng} / \mathrm{mL}$ to $134 \pm 5 \mathrm{ng} / \mathrm{mL}$ (77).

\section{Pycnogenol improves blood flow through nitric oxide-mediated vasorelaxation}

A central role of Pycnogenol is its ability to enhance endothelial production of NO from the substrate L-arginine, by the enzyme NOS. In an in vitro study it was shown that Pycnogenol, in a dose concentration range of $1-10 \mu \mathrm{g} / \mathrm{ml}$ relaxed epinephrine-, norepinephrine- or phenylephrine-induced contraction of the rat aorta. However, when the endothelial lining of the aortic ring was removed, Pycnogenol produced no effect, indicating an endothelium dependent relaxing (EDR) effect. This response was caused by enhanced NO levels, because the NOS inhibitor N-methyl-L arginine (NMA) reversed the relaxation, and this response in turn was reversed by addition of L-arginine. Pycnogenol-induced EDR persisted after exposure of intact rings to high levels of SOD, suggesting that the mechanism of EDR did not involve scavenging of superoxide anion (78). In addition to causing relaxation, preincubation of aortic rings with Pycnogenol $(1-10 \mu \mathrm{g} / \mathrm{ml})$ inhibited subsequent E- and NE-induced aortic contractions in a concentration dependent manner. Three fractions of the 
extract were used. It was interesting to note that fraction thee with oligomeric procyanidins exhibited potent EDR activity. It was concluded that Pycnogenol in addition to its known antioxidant activity stimulates constitutive endothelial NOS (eNOS) activity and increases the NO levels, which could counteract the vasoconstrictions induced by epinephrine and norepinephrine. Furthermore, additional protective effects could result from the well-known established properties of NO to decrease platelet aggregation and adhesions (79), as well as to inhibit LDL cholesterol oxidation (80). All these could protect against atherogenesis and thrombus formation, known risk factors in $\mathrm{CV}$ (78). These findings led to the proposition that Pycnogenol stimulates production of NO from L-arginine by eNOS (16). It remains to be tested whether Pycnogenol may influence the release of vasoactive substances from perivascular adipose tissue (see 81).

\section{Pycnogenol normalizes blood platelet activity}

The activation of platelets represents an important contributory factor in the pathogenesis of thrombosis (82). The effects of Pycnogenol supplementation were assessed on platelet function of cigarette smokers. In this population, a two fold increase of adrenaline levels and enhanced platelet activity is observed. Smoking is a well defined cardiovascular disease risk factor as already stated above. The increased platelet reactivity and aggregation observed 2 hours after smoking were inhibited by a single dose of either $500 \mathrm{mg}$ of acetyl salicylic acid or $100 \mathrm{mg}$ of Pycnogenol. This inhibitory effect on platelet reactivity/aggregation followed a clear dose response relationship, starting from a dose of $25 \mathrm{mg}$ to complete absence of platelet aggregation observed $200 \mathrm{mg}$ of Pycnogenol, the effect persisting for 6 days (83). Likewise, heavy smokers were given Pycnogenol at a daily dose of $200 \mathrm{mg}$ for 8 weeks. The platelet aggregation index was significantly reduced at the end of the experiment; a comparison with a normal non-smoker population showed that the platelet reactivity was almost reduced to the normal values of a control healthy population. Pycnogenol administration lowered the level of TxB2, a powerful vasoconstrictor and platelet aggregating agent (84).

\section{CONCLUSION}

Pycnogenol protects vascular endothelium, decreases capillary fragility, increase capillary resistance thus reduces vascular permeability, contributing to reduction of edema. It prevents thrombus formation through inhibiting platelet aggregation, improves microcirculatory function. It protects collagen degradation and increases its regeneration and produces capillary sealing effect through its specific binding capacity with collagen. All these activities of Pycnogenol contribute to its beneficial effects in CVI as a food supplement.

Developing new combination products using synergistic approaches are the future trends of research and development to prove efficacy in conditions which involves oxidative stress, inflammation, endothelial dysfunction, thrombogenesis, and ulceration.

\section{REFERENCES}

1. Mariman EC. Nutrigenomics in perspective. Pharmacogenomics 2007;8: 421-424.

2. Maritime Pine Extract. United States Pharmacopoeia National Formulary. Volume USP 30 - NF 25. Rockville: United States Pharmacopeial Convention, Inc; 2007. p 965-966.

3. Borzelleca JF, Burdock GA, Thomas JA. Opinion of an expert panel on the Generally Recognized As Safe (GRAS) status of French maritime pine bark extraxt (Pycnogenol(R)) as a flavoring agent. Internal document 2003.

4. Rohdewald PJ. Pycnogenol ${ }^{\circledR}$, French Maritime Pine Bark Extract. Encyclopedia of Dietary Supplements. New York: Marcel Dekker Inc., 2005. p 545-553.

5. Drehsen G. From ancient pine bark uses to Pycnogenol. In: Packer L, Hiramatsu M, Yoshikawa T, editors. Antioxidant Food Supplements in Human Health. Academic Press; 1999. p 311-322.

6. Gulati OP. The nutraceutical Pycnogenol: its role in cardiovascular health and blood glucose control. Biomed Rev 2005;16:49-57.

7. Arcangeli P. Pycnogenol in chronic venous insufficiency. Fitoterapia 2000;71:236-244.

8. Petrassi C, Mastromarino A, Spartera C. Pycnogenol in chronic venous insufficiency. Phytomedicine 2000;7:383388.

9. Koch R. Comparative study of Venostasin and Pycnogenol in chronic venous insufficiency. Phytother Res 2002;16 (Suppl 1):S1-5.

10. Schmidtke I, Schoop W. Le pycnogénol: Thérapeutique médicamenteuse de l'oedème statique. J Suisse Méd Globale 1995;3:114-115.

11. Schmidtke I, Schoop W. Das hydrostatische Ödem und seine medikamentöse Beeinflussung. Swiss Med 1984;6:67-69. 
12. Becker SR. Le pycnogénol: une substance douée de properiétés angioprotectrices dans le traitement de l'insuffisance veineuse chronique. J Suisse Méd Globale 1995;95:11-14.

13. Cesarone MR, Belcaro G, Rohdewald P, Pellegrini L, Ledda A, Vinciguerra G, et al. Comparison of Pycnogenol and Daflon in treating chronic venous insufficiency: a prospective, controlled study. Clin Appl Thromb Hemost 2006;12:205-212.

14. Vinciguerra G, Belcaro G, Cesarone MR, Rohdewald P, Stuard S, Ricci A, et al. Cramps and muscular pain: prevention with pycnogenol in normal subjects, venous patients, athletes, claudicants and in diabetic microangiopathy. Angiology 2006;57:331-339.

15. Gulati OP. Pycnogenol ${ }^{\circledR}$ in venous disorders: A review. Eur Bull Drug Res 1999;7: 8-13.

16. Rohdewald P. A review of the French maritime pine bark extract (Pycnogenol), a herbal medication with a diverse clinical pharmacology. Int J Clin Pharmacol Ther 2002;40:158-168.

17. Blumenthal M. Pycnogenol (French Maritime Pine Bark Extract) Pinus Pinaster Aiton subsp. Atlantica. The American Botanical Council guide to Herbs; 2003. p 369-373.

18. Wenner A, Leu HJ, Spycher MA, Brunner U. Ultrastructural changes of capillaries in chronic venous insufficiency. Exp Cell Biol 1980;48:1-14.

19. Bollinger A, Jager K, Geser A, Sgier F, Seglias J. Transcapillary and interstitial diffusion of Na-fluorescein in chronic venous insufficiency with white atrophy. Int $J$ Microcirc Clin Exp 1982;1:5-17.

20. Nicolaides AN. Investigation of chronic venous insufficiency: A consensus statement (France, March 5-9, 1997). Circulation 2000;102:E126-163.

21. Stucker M, Falkenberg M, Reuther T, Altmeyer P, Lubbers DW. Local oxygen content in the skin is increased in chronic venous incompetence. Microvasc Res 2000;59:99106.

22. Flore R, Gerardino L, Santoliquido A, Pola R, Flex A, Di Campli C, Pola P, Tondi P. Enhanced oxidative stress in workers with a standing occupation. Occup Environ Med 2004;61:548-550.

23. Nordmann H, Gulati OP. Acute venous stasis and chronic venous insufficiency models in rats. Methods Find Exp Clin Pharmacol 1983;5:347-355.

24. Nordmann H, Gulati OP. Hemodynamic studies in acute venous stasis edema in rats. Experientia 1984;40(4):346-8.
25. Gulati OP, Sharma SC, Hammersen F. Hemodynamic, biochemical and morphological changes in the dextran and D-galactosamine-induced edemas in rats. Arch Int Pharmacodyn Ther 1983;263(2):272-87.

26. Gulati OP, Malmsten C, Ponard G, Gulati N. The local edemogenic effects of leukotriene $\mathrm{C} 4$ and prostaglandin E2 in rats. Prostaglandins Leukot Med 1983;10:11-17.

27. Nordmann HJ, Dumont JM, Gulati OP, Sharma SC. Wirkungen von Venoruton ${ }^{\circledR}$ am Modell der akuten venösen Stauung an der Ratte. Swiss Med 1984;6:63-66.

28. Sharma SC, Gulati OP. Role of calcium in the histamine release induced by D-galactosamine from rat mast cells. Experientia 1985;41:1177-1178.

29. Belkhiri A, Richards C, Whaley M, McQueen SA, Orr FW. Increased expression of activated matrix metalloproteinase- 2 by human endothelial cells after sublethal H2O2 exposure. Lab Invest 1997;77:533-539.

30. Widmer LK, Stahelin HB, Nissen C, da Silva A. VenenArterienkrankheiten, Koronare Herzkrankheit bei Berufstätigen. Bern, Stuttgart, Wien: Verlag Hans Huber; 1981.

31. Leu AJ, Leu HJ, Franzeck UK, Bollinger A. Microvascular changes in chronic venous insufficiency - a review. Cardiovasc Surg 1995;3:237-245.

32. Junger M, Steins A, Hahn M, Hafner HM. Microcirculatory dysfunction in chronic venous insufficiency (CVI). Microcirculation 2000;7:S3-12.

33. Wollina U, Abdel-Naser MB, Mani R. A review of the microcirculation in skin in patients with chronic venous insufficiency: the problem and the evidence available for therapeutic options. Int J Low Extrem Wounds 2006;5:169180.

34. Junger M, Hahn M, Klyscz T, Steins A. Microangiopathy in the pathogenesis of chronic venous insufficiency. Curr Probl Dermatol 1999;27:124-129.

35. Bergan JJ, Schmid-Schonbein GW. Therapeutic management of chronic venous insufficiency: microcirculation as a target. Microcirculation 2000;7(6 Pt 2):S23-28.

36. Virgili F, Kim D, Packer L. Procyanidins extracted from pine bark protect alpha-tocopherol in ECV 304 endothelial cells challenged by activated RAW 264.7 macrophages: role of nitric oxide and peroxynitrite. FEBS Lett 1998;431:315-318.

37. Packer L, Rimbach G, Virgili F. Antioxidant activity and biologic properties of a procyanidin-rich extract from pine (Pinus maritima) bark, Pycnogenol. Free Radic Biol Med 1999;27:704-724. 
38. Virgili F, Kobuchi H, Noda Y, Cossins E, Packer L. Procyanidins from Pinus maritima bark: Antioxidant activity, effects on the immune system and modulation of nitrogen monoxide metabolism. In: Packer L, Hiramatsu M, Yoshikawa T, editors. Antioxidant Food Supplements in Human Health. Academic Press; 1999. p 323-342.

39. Lau BHS, Riesen SK, Truong KP, Lau EW, Rohdewald P, Barreta RA. Pycnogenol ${ }^{\circledR}$ as an adjunct in the management of childhood asthma. J Asthma 2004;41:825-832.

40. Blazso G, Gabor M, Sibbel R, Rohdewald P. Anti-inflammatory and superoxide radical scavenging activities of procyanidins containing extract from the bark of Pinus pinaster sol. and its fractions. Pharm Pharmacol Lett 1994;3:217-220.

41. Rong Y, Li L, Shah V, Lau BH. Pycnogenol protects vascular endothelial cells from t-butyl hydroperoxide induced oxidant injury. Biotechnol Ther 1995;5:117-126.

42. Noda Y, Anzai K, Mori A, Kohno M, Shinmei M, Packer L. Hydroxyl and superoxide anion radical scavenging activities of natural source antioxidants using the computerized JES-FR30 ESR spectrometer system. Biochem Mol Biol Int 1997;42:35-44.

43. Nelson AB, Lau BH, Ide N, Rong Y. Pycnogenol inhibits macrophage oxidative burst, lipoprotein oxidation, and hydroxyl radical-induced DNA damage. Drug Dev Ind Pharm 1998;24:139-144.

44. Chida M, Suzuki K, Nakanishi-Ueda T, Ueda T, Yasuhara $\mathrm{H}$, Koide $\mathrm{R}$, et al. In vitro testing of antioxidants and biochemical end-points in bovine retinal tissue. Ophthalmic Res 1999;31: 407-415.

45. Cossins E, Lee R, Packer L. ESR studies of vitamin C regeneration, order of reactivity of natural source phytochemical preparations. Biochem Mol Biol Int 1998;45:583597.

46. Kim J CJ, Pinnas JL, Mooradian AD. Effect of selected antioxidants on malondialdehyde modification of proteins. Nutrition 2000;16:1079-1081.

47. Peng Q, Wei Z, Lau BH. Pycnogenol inhibits tumor necrosis factor-alpha-induced nuclear factor kappa B activation and adhesion molecule expression in human vascular endothelial cells. Cell Mol Life Sci 2000;57:834-841.

48. Sharma SC, Sharma S, Gulati OP. Pycnogenol prevents haemolytic injury in G6PD deficient human erythrocytes. Phytother Res 2003;17:671-674.

49. Bayeta E BM, Lau HS. Pycnogenol inhibits generation of inflammatory mediators in macrophages. Nutr Res
2000;20:249-259.

50. Wei ZH, Peng Q, Lau B. Pycnogenol ${ }^{\odot}$ enhances endothelial cell antioxidant defenses. Redox Report 1997;3:219-224.

51. Saliou C, Rimbach G, Moini H, McLaughlin L, Hosseini S, Lee J, et al. Solar ultraviolet-induced erythema in human skin and nuclear factor-kappa-B-dependent gene expression in keratinocytes are modulated by a French maritime pine bark extract. Free Radic Biol Med 2001;30:154-160.

52. Sime S, Reeve VE. Protection from inflammation, immunosuppression and carcinogenesis induced by UV radiation in mice by topical Pycnogenol. Photochem Photobiol 2004;79:193-198.

53. Blazso G, Rohdewald P, Sibbel R, Gabor M. Anti-inflammatory activities of procyanidin-containing extracts from Pinus pinaster sol. In: Antus S, Gabor M, Vetschera K, editors; 1995 July 16-19; Vienna, Austria. p 231-238.

54. Blazso G, Gabor M, Rohdewald P. Antiinflammatory activities of procyanidin-containing extracts from Pinus pinaster Ait. after oral and cutaneous application. Pharmazie 1997;52:380-382.

55. Cho KJ, Yun CH, Packer L, Chung AS. Inhibition mechanisms of bioflavonoids extracted from the bark of Pinus maritima on the expression of proinflammatory cytokines. Ann N Y Acad Sci 2001;928:141-156.

56. Grimm T, Schafer A, Hogger P. Antioxidant activity and inhibition of matrix metalloproteinases by metabolites of maritime pine bark extract (pycnogenol). Free Radic Biol Med 2004;36:811-822.

57. Sharma SC, Sharma S, Gulati OP. Pycnogenol inhibits the release of histamine from mast cells. Phytother Res 2003;17:66-69.

58. Blazso G, Gabor M, Schonlau F, Rohdewald P. Pycnogenol accelerates wound healing and reduces scar formation. Phytother Res 2004;18:579-581.

59. Belcaro G, Cesarone MR, Errichi BM, Ledda A, Di Renzo A, Stuard S, et al. Venous ulcers: microcirculatory improvement and faster healing with local use of Pycnogenol. Angiology 2005;56:699-705.

60. Maritim A, Dene BA, Sanders RA, Watkins JB, 3rd. Effects of pycnogenol treatment on oxidative stress in streptozotocin-induced diabetic rats. J Biochem Mol Toxicol 2003;17:193-199.

61. Berryman AM, Maritim AC, Sanders RA, Watkins JB, 3rd. Influence of treatment of diabetic rats with combinations of pycnogenol, beta-carotene, and alpha-lipoic acid on parameters of oxidative stress. $J$ Biochem Mol Toxicol 
2004;18:345-352.

62. Dene BA, Maritim AC, Sanders RA, Watkins JB, 3rd. Effects of antioxidant treatment on normal and diabetic rat retinal enzyme activities. $J$ Ocul Pharmacol Ther 2005;21:28-35.

63. Devaraj S, Vega-Lopez S, Kaul N, Schonlau F, Rohdewald P, Jialal I. Supplementation with a pine bark extract rich in polyphenols increases plasma antioxidant capacity and alters the plasma lipoprotein profile. Lipids 2002;37:931934.

64. Durackova Z, Trebaticky B, Novotny V, Zitnanova I, Breza J. Lipid metabolism and erectile function improvement by Pycnogenol, extract from the bark of Pinus pinaster in patients suffering from erectile dysfunction- a pilot study. Nutr Res 2003;23:1189-1198.

65. Segger D, Schonlau F. Supplementation with Evelle improves skin smoothness and elasticity in a double-blind, placebo-controlled study with 62 women. $J$ Dermatolog Treat 2004;15:222-226.

66. Ni Z, Mu Y, Gulati O. Treatment of melasma with Pycnogenol. Phytother Res 2002;16:567-71.

67. Roseff SJ. Improvement in sperm quality and function with French maritime pine tree bark extract. $J$ Reprod Med 2002;47:821-824.

68. Kohama T, Suzuki N, Ohno S, Inoue M. Analgesic efficacy of French maritime pine bark extract in dysmenorrhea: an open clinical trial. J Reprod Med 2004;49: 828-832.

69. Kimbrough C, Chun, M., Dela Roca, G., Lau BHS. Pycnogeno ${ }^{\mathbb{O}}$ chewing gum minimizes gingival bleeding and plaque formation. Phytomedicine 2002;9: 410-413.

70. Cesarone MR, Belcaro G, Rohdewald P, Pellegrini L, Ippolito E, Scoccianti M, et al. Prevention of edema in long flights with Pycnogenol. Clin Appl Thromb Hemost 2005;11:289-294.

71. Belcaro G, Cesarone MR, Rohdewald P, Ricci A, Ippolito $\mathrm{E}$, Dugall $\mathrm{M}$, et al. Prevention of venous thrombosis and thrombophlebitis in long-haul flights with pycnogenol. Clin Appl Thromb Hemost 2004;10:373-377.

72. Kohama T. Nutritional supplements in clinical practice. Prog Med 2004;24:1503-1510.

73. Scurr JH, Gulati OP. Zinopin ${ }^{\circledR}$ - the rationale of its use as a food supplement in traveller's thrombosis and motion sickness. Phytother Res 2004;18:687-695.

74. Scurr JH, Gulati OP. Zinopin ${ }^{\circledR}$ - its use as a food supplement in traveller's thrombosis, oedema and motion sickness. Eur Bull Drug Res 2005;13:77-81.

75. Cesarone MR, Belcaro G, Rohdewald P, Pellegrini L, Ledda A, Vinciguerra G, et al. Rapid relief of signs/symptoms in chronic venous microangiopathy with pycnogenol: a prospective, controlled study. Angiology 2006;57:569576.

76. Araghi-Niknam M, Hosseini S, Larson D, Rohdewald P, Watson RR. Pine bark extract reduces platelet aggregation. Integr Med 2000;2:73-77.

77. Hosseini S, Lee J, Sepulveda RT, Rohdewald P, Watson RR. A randomized, double-blind, placebo-controlled, prospective, 16 week crossover study to determine the role of Pycnogenol in modifying blood pressure in mildly hypertensive patients. Nutr Res 2001;21:1251-1260.

78. Fitzpatrick DF, Bing B, Rohdewald P. Endotheliumdependent vascular effects of Pycnogenol. J Cardiovasc Pharmacol 1998;32:509-515.

79. Yao SK, Ober JC, Krishnaswami A, Ferguson JJ, Anderson HV, Golino P, et al. Endogenous nitric oxide protects against platelet aggregation and cyclic flow variations in stenosed and endothelium-injured arteries. Circulation 1992;86:1302-1309.

80. Hogg N, Kalyanaraman B, Joseph J, Struck A, Parthasarathy S. Inhibition of low-density lipoprotein oxidation by nitric oxide. Potential role in atherogenesis. FEBS Lett 1993;334:170-174.

81. Chaldakov GN. Cardiovascular adipobiology: a novel. Heart-associated adipose tissue in cardiovascular disease. Ser J Exp Clin Res 2008; 9: 81-88.

82. Watson RR. Reduction of cardiovascular disease risk factors by French Maritime Pine Bark Extract. Cardiovasc Rev Reports 1999; 20:326-329.

83. Putter M, Grotemeyer KH, Wurthwein G, Araghi-Niknam M, Watson RR, Hosseini S, et al. Inhibition of smokinginduced platelet aggregation by aspirin and pycnogenol. Thromb Res 1999;95:155-161.

84. Araghi-Niknam M, Hosseini S, Larson D, Rohdewald P, Watson RR. Pine bark extract reduces platelet aggregation. Integr Med 1999;2:73-77. 